\title{
Panorama da Saúde Ocupacional existente no setor da Veterinária em Portugal (2017/8)
}

\section{OVERVIEW OF VETERINARY SECTOR OCCUPATIONAL HEALTH AT PORTUGAL (2017/8)}

TIPO DE ARTIGO: Observacional Analítico Transversal

AUTORES: Santos M(1), Almeida A(2), Lopes C(3), Oliveira T(4).

\section{RESUMO}

\section{Introdução/ enquadramento/ objetivos}

Os Médicos Veterinários e os profissionais que com eles colaboram (Enfermeiros Veterinários e Auxiliares Veterinários) estão sujeitos a inúmeros riscos ocupacionais. É objetivo deste estudo caraterizar a sua perceção relativa à existência de riscos e fatores de risco laborais, a utilização que fazem dos equipamentos de proteção individual, a sua suscetibilidade a acidentes de trabalho e doenças profissionais, a exposição ao stress laboral e o recurso à equipa de saúde ocupacional.

\section{Metodologia}

Trata-se de um estudo descritivo, correlacional, de carater transversal, realizado após a aplicação de um inquérito online, com recurso a uma técnica de amostragem por conveniência, que contou com a colaboração de associações, sindicatos, clínicas veterinárias, bem como algumas empresas de saúde ocupacional. A recolha de dados ocorreu entre junho de 2017 e março de 2018 e o valor final da amostra representa o número de Médicos, Enfermeiros e Auxiliares Veterinários que trabalham em clínicas/ hospitais veterinários que se prontificaram a responder anónima e voluntariamente ao inquérito.

\section{Resultados}

Responderam 91 indivíduos tendo sido validados 90 inquéritos (eliminou-se um pela incoerência das respostas). A amostra foi constituída maioritariamente por trabalhadores do sexo feminino $(87,8 \%)$, com idade inferior a 30 anos $(53,3, \%)$, com pouca experiência profissional (53,3\% trabalha há menos de 5 anos) e tendo como profissão predominante a medicina $(80,0 \%)$. A nível dos fatores de risco destacam-se o contacto com produtos 
imunoalergénicos, o stress, a manipulação de produtos químicos e o risco biológico no contacto com os animais. Relativamente aos equipamentos de proteção individual sobressaem o uso de farda e de máscara. A existência de história pessoal de acidentes de trabalho é muito frequente $(93,3 \%)$, sendo muito prevalentes os traumatismos infligidos pelos animais (como mordidelas, arranhadelas ou escoriações), seguido das picadas ou cortes com objetos cortantes potencialmente contaminados. No entanto, foram escassos os casos em que existiram limitações funcionais. A nível de doenças profissionais sobressaem as lesões músculo-esqueléticas $(26,7 \%)$, embora apenas um caso tenha sido declarado como suspeita de doença profissional. O stress emergiu como um fenómeno muito frequente, atingindo a quase totalidade dos trabalhadores $(96,7 \%)$, destacando-se a dificuldade em conciliar as vidas, profissional e pessoal $(81,1 \%)$, a insatisfação salarial $(76,7 \%)$ e a elevada carga de trabalho $(73,3 \%)$. Por fim, observa-se que a maioria $(63,2 \%)$ tem exame com a Medicina de Trabalho pelo menos de dois em dois anos, mas apenas $28,9 \%$ tem a perceção do trabalho realizado pelo Técnico de Higiene e Segurança e somente $26,7 \%$ refere ter tido formação relacionada com os seus riscos laborais.

\section{Conclusão}

Uma amostra pequena de 90 indivíduos divididos em três classes profissionais de caraterísticas diversas e questões que não conseguiram abarcar todas as respostas possíveis poderão ter enviesado os resultados do tratamento estatístico; pois se em algumas situações se encontraram relações entre variáveis concordantes com a bibliografia ocupacional para a generalidade das profissões e em sintonia com a experiência clínica dos autores, noutros casos ocorreu o oposto.

Entre as diversas relações estatísticas evidenciadas destacam-se as associações entre o sexo feminino, o stress e a existência genérica de sintomas associados ao trabalho; assim como as associações entre exercer como Médico Veterinário, sentir stress laboral por conflito com os clientes e estar exposto a acidentes por corte/ picada com objetos potencialmente contaminados.

Este estudo serviu como ponto de partida para uma primeira caraterização sumária deste setor, sendo obviamente desejável um estudo de maior dimensão, com questões mais abrangentes e melhor estruturadas.

Palavras-chave: saúde ocupacional, saúde do trabalhador e medicina do trabalho; medicina veterinária, médico veterinário, enfermagem veterinária, enfermeiro veterinário, auxiliar veterinário.

\section{ABSTRACT}

\section{Introduction / framework / objectives}

Veterinarians and their collaborators (Veterinarian Nurses and Auxiliaries) are subject to numerous occupational hazards. The objective of this study is to characterize their perception regarding the existence of occupational risk and risk factors, their use of 
personal protective equipment, susceptibility to occupational accidents and diseases, exposure to work stress and the use of occupational health team.

\section{Methodology}

This is a descriptive, cross-sectional study, carried out after the application of an online survey, using a sampling technique for convenience, with the collaboration of associations, veterinary clinics, as well as some companies of occupational health. Data collection took place between June 2017 and March 2018 and the final value of the sample represents the number of veterinarians, veterinary nurses and auxiliaries working in veterinary clinics / hospitals who have volunteered to respond anonymously and voluntarily to the survey.

\section{Results}

Ninety-one individuals responded and 90 surveys were validated (one was eliminated because of incoherence of responses). The sample consisted mainly of female workers $(87.8 \%)$, under the age of $30(53.3 \%)$, with little professional experience $(53.3 \%$ worked for less than 5 years) and predominant profession in veterinary medicine $(80.0 \%)$. Risk factors include contact with immunoallergenic products, stress, handling of chemicals and biological risk in contact with animals. The use of uniforms and a mask is particularly important for personal protective equipment. The existence of a personal history of occupational accidents is very frequent (93.3\%), and animal injuries such as nibbling, scratching or abrasions are very prevalent, followed by bites or cuts with potentially contaminated sharp objects. However, there were few cases where there were functional limitations. At the level of occupational diseases, the musculoskeletal lesions $(26.7 \%)$ stand out, although only one case has been declared as a suspected occupational disease. Stress emerged as a very frequent phenomenon, affecting almost all workers $(96.7 \%)$, especially the difficulty in reconciling professional and personal lives $(81.1 \%)$, wage dissatisfaction (76.7\%) and the high workload (73.3\%). Finally, it is observed that the majority $(63.2 \%)$ have a work medicine consultation at least every two years, but only $28.9 \%$ perceive the work performed by the Technician of Hygiene and Safety and only $26.7 \%$ report having workshops related to their occupational hazards.

\section{Conclusion}

A small sample of 90 individuals divided into three professional classes with distinctive characteristics and questions that failed to cover all the possible answers may have biased the results of the statistical treatment; because if in some situations relationships were found between variables consistent with the occupational bibliography for most of the professions and in line with the authors' clinical experience, in other cases the opposite occurred.

Among the several statistical relationships highlighted, the associations between female sex, stress and the general existence of symptoms associated with work, as well as the associations between exercising as a veterinarian, feeling occupational stress due to 
conflict with clients and being exposed to cut / chop accidents with potentially contaminated objects.

This study served as a starting point for a first summary characterization of this sector, and a larger study with broader and better structured questions is obviously desirable.

Words/ key expressions: occupational health, worker health, and occupational medicine; veterinary medicine, veterinary doctor, veterinary nurse, veterinary assistant.

\section{INTRODUÇÃO}

Os Médicos Veterinários e os profissionais que com eles colaboram (Enfermeiros e Auxiliares) estão sujeitos a inúmeros riscos ocupacionais; contudo, a bibliografia explícita nesta área é escassa e não foram encontradas guidelines orientadoras consensuais.

Resumindo uma revisão bibliográfica efetuada por uma das autoras destes trabalho, pode-se salientar, por exemplo, um estudo Australiano de 2005 que mencionou que 66\% dos acidentes nestes profissionais envolve trauma direto com os animais; a situação onde tal mais frequentemente acontecia era na imobilização dos mesmos para a realização de exame físico e exames auxiliares de diagnóstico, sobretudo nos de grande porte (situação dez vezes mais frequente). Deveriam ainda ser consideradas as mordeduras e arranhadelas, graves em alguns casos e os acidentes com agulhas e outros instrumentos corto-perfurantes. Uma pequena parte dos acidentes eram de viação, sobretudo quando o veterinário se deslocava à empresa cliente, para consultar animais de grande porte.

Tal como para os acidentes de trabalho, não abundavam estatísticas relativas a doenças profissionais neste setor. Neste contexto destacavam-se o eczema de contato alérgico ao pelo, penas e/ ou secreções dos animais, ao látex (sendo que o uso de luvas sem pó atenua a situação) e a fármacos (como antibióticos e anestésicos); bem como asma ocupacional e, obviamente, as zoonoses. Estudos sobre mutagenicidade e teratogenicidade são, geralmente, controversos ou inconclusivos, existindo ainda assim destaque neste contexto para os anestésicos, citostáticos e as radiações ionizantes.

\section{METODOLOGIA}

Trata-se de um estudo descritivo, correlacional, de carater transversal.

A amostragem foi por conveniência e o valor final da amostra representa o número de Médicos, Enfermeiros e Auxiliares Veterinários que trabalham em clínicas/ hospitais e que se prontificaram a responder voluntariamente ao inquérito.

Para a recolha de dados optou-se por um inquérito online; para o efeito obteve-se a colaboração institucional da Associação Portuguesa de Médicos Veterinários Especialistas em Animais de Companhia que disponibilizou o acesso ao questionário aos 
seus associados. Foram ainda realizados contatos com instituições, sindicatos, clínicas veterinárias e revistas que tivessem associados inseridos nestas áreas, bem como empresas de saúde ocupacional por forma a que divulgassem o estudo e o link de acesso aos interessados. A recolha de dados ocorreu entre junho de 2017 e março de 2018.

No total, foram operacionalizadas diversas questões para dar resposta a diferentes domínios que se pretendiam conhecer: 1-Riscos e fatores de risco laborais; 2Equipamentos de Proteção Individual utilizados; 3-Acidentes de trabalho; 4-Doenças profissionais; 5-Exposição ao stress laboral; 6-Recurso à equipa de saúde ocupacional.

A normalidade da distribuição de cada variável foi avaliada por meio do teste de Kolmogorov-Smirnov. No tratamento estatístico foram utilizadas medidas de tendência central para descrever os achados. Para a análise inferencial, usaram-se os testes do Qui quadrado, Exato de Fisher, coeficiente V de Cramer, Correlação de Spearman, U de Mann-Whitney e Kruskall-wallis, para análise de variáveis categóricas e/ou nominais. Para a análise de variáveis discretas, utilizou-se o teste $\mathrm{T}$. A relação entre as variáveis será dada pelo valor de $p$. Para valores de $p<0.05$ rejeita-se a hipótese nula, ou seja, a probabilidade de as diferenças registadas na amostra serem devidas ao acaso é muito pequena. Caso o p>0,05, considera-se que não existe evidência suficiente para rejeitar a hipótese nula. Os dados foram processados com a ajuda do SPSS $_{24}$.

\section{APRESENTAÇÃO DE RESULTADOS E DISCUSSÃO}

\section{Caraterização sociodemográfica}

A caraterização sociodemográfica da amostra pode ser consultada na Tabela 1. As regras da RPSO recomendam que os quadros e tabelas sejam inseridos no final do artigo mas, dado o número elevado destas estruturas neste trabalho, os autores entenderam que o informação seria mais facilmente entendida casos inserissem estas unidades ao longo do texto e não no final, todas juntas.

Os números demonstram uma população trabalhadora muito jovem, tendo a maioria $(53,3 \%)$ uma idade inferior a 30 anos. Contudo, esta distribuição etária poderá não refletir com rigor a realidade, mas sim estar influenciada pelo eventual maior à vontade que indivíduos mais jovens têm perante o uso de computador, internet e inquéritos on line.

É uma amostra predominantemente feminina $(87,8 \%)$, donde se destaca a disponibilidade dos Médicos Veterinários para responderem ao questionário ( $80 \%)$, facto que talvez demonstre uma maior consciencialização desta classe face aos riscos laborais, uma vez que, nas clínicas veterinárias não é usual existirem Médicos, Enfermeiros ou Auxiliares nestas proporções entre si. 
Em relação à experiência profissional, ou seja, número de anos a exercer nesta área, o grupo menos experiente (com menos de 5 anos de trabalho) foi o mais responsivo $(53,4 \%)$, indo de encontro aos valores já revelados para a faixa etária, facto que também ajuda a corroborar a hipótese de que os mais jovens estão mais propensos a utilizar as novas tecnologias, predispondo-se, sem dificuldade, a responder a este tipo de questionários.

Tabela 1- Caraterização sociodemográfica da amostra

Frequência Percentagem (\%)

\begin{tabular}{|c|c|c|c|}
\hline \multirow[t]{4}{*}{ Idade } & $<20$ anos & 1 & 1,1 \\
\hline & 21 a 30 & 47 & 52,2 \\
\hline & 31 a 40 & 33 & 36,7 \\
\hline & 41 a 50 & 9 & 10,0 \\
\hline \multirow[t]{2}{*}{ Sexo } & Feminino & 79 & 87,8 \\
\hline & Masculino & 11 & 12,2 \\
\hline \multirow[t]{3}{*}{ Profissão } & Médico Veterinário & 72 & 80,0 \\
\hline & Enfermeiro Veterinário & 13 & 14,4 \\
\hline & Ajudante/ auxiliar & 5 & 5,6 \\
\hline \multirow[t]{4}{*}{ Experiência profissional } & Menos que um ano & 8 & 8,9 \\
\hline & 1 a 5 anos & 40 & 44,4 \\
\hline & 6 a 10 anos & 21 & 23,3 \\
\hline & Mais que 10 anos & 21 & 23,3 \\
\hline
\end{tabular}

\section{Riscos/ fatores de risco laborais}

Assinalando alguns riscos/ fatores de risco laborais, foi pedido aos participantes que os classificassem numa escala de importância (ponderando a frequência e a gravidade) ver Tabela 2.

É de salientar que os inquiridos demonstraram genericamente valorizar os seus riscos laborais, pois em cinco dos oito itens avaliados, não houve um único indivíduo que tenha considerado que tal risco era nulo; acrescenta-se ainda que nos restantes parâmetros, a percentagem que respondeu nulo foi diminuta.

Inversamente, a consideração de risco elevado obtém a maior frequência para todos os fatores de risco/ riscos assinalados, exceto os citostáticos (talvez porque efetivamente só um grupo restrito de profissionais terá de equacionar este parâmetro). 
Tabela 2 - Auto perceção da frequência e gravidade de alguns riscos/ fatores de risco laborais

Fator de risco/ risco
Valorização da importância

(frequência versus gravidade) - (\%)

\begin{tabular}{lllll} 
& Nula & Pequena & Moderada & Elevada \\
\cline { 2 - 5 } $\begin{array}{l}\text { Produtos imunoalérgicos (urina, fezes, } \\
\text { penas, latex...) }\end{array}$ & 0 & 4,4 & 14,4 & 81,1 \\
\hline $\begin{array}{l}\text { Substâncias capazes de causar infeções } \\
\text { (zoonoses) }\end{array}$ & 0 & 6,7 & 31,1 & 62,2 \\
\hline $\begin{array}{l}\text { Agentes químicos (fármacos, detergentes, } \\
\text { esterilizantes) }\end{array}$ & 0 & 4,4 & 21,1 & 74,4 \\
\hline Esforço físico/ cargas & 0 & 12,2 & 43,3 & 44,4 \\
\hline Posturas forçadas/ mantidas & 1,1 & 11,1 & 32,2 & 55,6 \\
\hline Risco cancerígeno (citostáticos e rx) & 6,7 & 36,7 & 26,7 & 30,0 \\
\hline Stress & 0 & 4,4 & 17,8 & 77,8 \\
\hline Outros & 27,8 & 20 & 26,7 & 25,6
\end{tabular}

Infere-se que a perceção de risco possa ser, eventualmente, mais elevada face à generalidade das profissões devido ao fato de a maioria dos que responderam terem um nível académico superior e serem profissionais associados à saúde. Por outro lado, a confrontação com as perguntas do inquérito pode também ter aumentado a consciencialização face aos riscos das tarefas profissionais.

Constata-se ainda que cerca de três quartos dos inquiridos considerou ter outros riscos/ fatores de risco não inseridos na listagem elaborada, 25,6\% dos quais de elevada gravidade. Assim, teria sido muito importante ter acrescentado uma questão aberta para que se pudessem conhecer e classificar esses fatores.

Analisando as relações entre as caraterísticas sociodemográficas e o número de fatores de risco elencados pela amostra, constata-se que não existem relações estatisticamente significativas (Tabela 3).

Tabela 3- Relação entre as caraterísticas sociodemográficas e o número de fatores de risco elencados

\begin{tabular}{llllll} 
& n & Média & $\begin{array}{c}\text { Desvio } \\
\text { Padrão }\end{array}$ & Teste \\
\hline Idade & Inferior a 30 anos & 48 & 7,71 & 0,459 & $\begin{array}{r}(t=1,106 ; \\
p=0,272)\end{array}$ \\
\cline { 2 - 4 } & & & & &
\end{tabular}


Superior ou igual a $30 \quad 42 \quad 7,57 \quad 0,703$

anos

\begin{tabular}{|c|c|c|c|c|c|}
\hline \multirow[t]{2}{*}{$\begin{array}{l}\text { Experiência } \\
\text { profissional }\end{array}$} & $\begin{array}{l}\text { Até } 5 \text { anos de } \\
\text { experiência }\end{array}$ & 48 & 7,69 & 0,512 & \multirow[t]{2}{*}{$\begin{array}{l}(t=0,743 ; \\
p=0,460)\end{array}$} \\
\hline & $\begin{array}{l}\text { Mais de } 5 \text { anos de } \\
\text { experiência }\end{array}$ & 42 & 7,60 & 0,665 & \\
\hline \multirow[t]{2}{*}{ Sexo } & Feminino & 79 & 7,62 & 0,606 & \multirow{2}{*}{$\begin{array}{l}(t=-1,417 \\
p=0,175)\end{array}$} \\
\hline & Masculino & 11 & 7,82 & 0,405 & \\
\hline \multirow[t]{3}{*}{ Profissão } & Médico Veterinário & 72 & 7,68 & 0,601 & \multirow{3}{*}{$\begin{array}{l}(F=0,779 \\
0,462)\end{array}$} \\
\hline & Enfermeiro Veterinário & 13 & 7,54 & 0,519 & \\
\hline & Auxiliar & 5 & 7,40 & 0,548 & \\
\hline
\end{tabular}

Relativamente à idade e à experiência profissional, esperava-se que um maior número de anos de trabalho implicasse uma maior capacidade para identificar os fatores de riscos e riscos laborais. Por outro lado, em função da personalidade e eventos de vida, tal também pode ter contribuído para banalizar os riscos.

Observa-se também que, em média, os indivíduos do sexo masculino identificaram um maior número de fatores de risco o que, por questões de personalidade e sobretudo culturais, normalmente não costuma acontecer, sendo expectável que demonstrem valorizar menos questões associadas à saúde, ou pelo menos, estar menos à vontade para o demonstrar publicamente.

No que diz respeito à gravidade com que os diferentes fatores de risco são percecionados, o teste de correlação de Spearman não revelou correlações estatisticamente significativas com a idade ou a experiência profissional, resultado idêntico ao encontrado para o sexo após aplicar o coeficiente $\mathrm{V}$ de Cramer. Relativamente à profissão, o Teste Exato de Fisher revelou algumas diferenças estatisticamente significativas associadas à perceção da intensidade/ gravidade dos riscos laborais, das quais se destacam a maior valorização por parte dos médicos veterinários do contato com produtos imunoalérgicos $\left(X^{2}=9,220 ; p=0,030\right)$.

\section{Equipamentos de proteção individual (EPIs)}

Quanto à existência de equipamentos de proteção individual (EPIs) e à frequência de uso dos mesmos, destaca-se a farda, utilizada pela grande maioria dos indivíduos, seguido da máscara e dos protetores de calçado. Em sentido oposto encontram-se os manguitos, as galochas e a viseira, pouco valorizados no decorrer das tarefas profissionais (Tabela 4). É ainda de realçar que, por lapso informático, não constava no inquérito final disponibilizado a opção luvas de proteção individual; no entanto, acreditase que, à semelhança da farda, teria grande expressividade em termos de percentagem de respostas. 
Tabela 4- Equipamentos de proteção individual- tipo e frequência de utilização na amostra

\begin{tabular}{lllll} 
EPls & $\begin{array}{l}\text { Não } \\
\text { tenho }\end{array}$ & $\begin{array}{l}\text { Tenho mas } \\
\text { não uso }\end{array}$ & $\begin{array}{l}\text { Tenho mas uso } \\
\text { pouco }\end{array}$ & $\begin{array}{l}\text { Tenho e uso com } \\
\text { frequência }\end{array}$ \\
\hline Farda & 0 & 0 & 1,1 & 98,9 \\
\hline Manguitos & 77,8 & 11,1 & 6,7 & 4,4 \\
\hline Viseira & 72,2 & 10,0 & 13,3 & 4,4 \\
\hline Máscara & 14,4 & 10,0 & 42,2 & 33,3 \\
\hline $\begin{array}{l}\text { Proteção de } \\
\text { calçado }\end{array}$ & 57,8 & 12,2 & 21,1 & 8,9 \\
\hline Galochas & 76,7 & 6,7 & 2,2 & 14,4
\end{tabular}

Relacionando o uso dos diferentes EPIs com a idade, o teste de Fisher não revelou diferenças estatisticamente significativas. Tal poderá ser explicado em função dos conhecimentos/ experiência adquirida, personalidade e dos acontecimentos de vida; pressupõe-se que os riscos profissionais possam ficar mais ou menos valorizados à medida que a idade avança, estando o uso de EPIs condicionado a essas crenças.

Em situação semelhante está a experiência profissional e a profissão. Seria expetável que o aumento da experiência implicasse uma maior valorização dos fatores de risco/ riscos profissionais e que tal se refletisse no uso mais frequente dos EPIs. De igual forma está a profissão pois, tal como é descrito na literatura, seria expetável que indivíduos mais diferenciados academicamente usassem EPIs com maior regularidade. No entanto, em função de outras variáveis, como a personalidade, talvez também possa acontecer que alguns funcionários se sintam mais confiantes e à vontade com o seu trabalho, a ponto de não sentirem necessidade de proteção.

Por último, utilizando o coeficiente $\vee$ de Cramer, não foi possível encontrar relações significativas com a variável sexo. No entanto, ao analisar a utilização de EPIs, individualmente, observa-se que o uso de manguitos foi estatisticamente mais prevalente no sexo masculino ( $V$ Cramer $=0,418, p=0,008$ ), assim como o uso de protetores de calçado (V Cramer $=0,253 ; p=0,138)$ e de galochas (V Cramer $=0,244 ; p=0,133)$ embora estes dois sem significância estatística.

\section{Acidentes de trabalho}

Cerca de 93,3\% dos inquiridos afirmaram já ter tido algum acidente de trabalho enquanto profissionais de Veterinária, destacando-se os traumatismos infligidos pelos animais, como mordidelas, arranhadelas ou escoriações; seguido das picadas ou cortes com objetos cortantes potencialmente contaminados. Mais uma vez se constata a ausência de uma pergunta aberta, no questionário, para especificar o tipo de acidentes representados no item "Outros", referenciado por cerca de $20 \%$ da amostra (Gráfico 1). 
Investigando a eventual relação entre a idade, o sexo e a experiência profissional com o número de acidentes de trabalho de cada indivíduo, o Teste $T$ revelou que não existiram relações estatisticamente significativas, ainda que em média os profissionais do sexo masculino, mais velhos e/ou mais experientes tenham tido mais acidentes (Tabela 5). Realça-se, no entanto, que mais idade, em princípio, implica mais anos a trabalhar, pelo que o número de acidentes no total poderá facilmente ser superior ao de funcionários mais jovens, com menos anos de trabalho. Para além disso, enquanto ser mais jovem e inexperiente poderá proporcionar atitudes mais precipitadas conduzindo à ocorrência de acidentes, ser mais velho e experiente, poderá proporcionar uma sensação de segurança, levando à não adoção de algumas medidas de proteção individual ou coletiva. Relativamente ao sexo, classicamente, a literatura descreve que o sexo feminino costuma ser mais cumpridor em relação ao uso dos EPIs, ainda que geralmente também tenham tarefas laborais com riscos menos frequentes e/ou graves, logo, a necessitar menos de EPIs. Simultaneamente, o sexo masculino tem tendência para ser mais impulsivo e/ou agressivo, logo, mais propenso a acidentes mais frequentes e/ou graves.

Tabela 5- Relação entre as caraterísticas sociodemográficas e o número de acidentes de trabalho

\begin{tabular}{|c|c|c|c|c|c|}
\hline & & $\mathbf{n}$ & Média & $\begin{array}{l}\text { Desvio } \\
\text { Padrão }\end{array}$ & Testes \\
\hline \multirow[t]{2}{*}{ Idade } & Inferior a 30 anos & 48 & 1,79 & 0,771 & \multirow{2}{*}{$\begin{array}{l}(t=-1,833 ; \\
p=0,070)\end{array}$} \\
\hline & $\begin{array}{l}\text { Superior ou igual a } 30 \\
\text { anos }\end{array}$ & 42 & 2,07 & 0,677 & \\
\hline \multirow[t]{2}{*}{$\begin{array}{l}\text { Experiência } \\
\text { profissional }\end{array}$} & $\begin{array}{l}\text { Até } 5 \text { anos de } \\
\text { experiência }\end{array}$ & 48 & 1,79 & 0,771 & \multirow[t]{2}{*}{$\begin{array}{l}(t=-1,833 ; \\
p=0,070)\end{array}$} \\
\hline & $\begin{array}{l}\text { Mais de } 5 \text { anos de } \\
\text { experiência }\end{array}$ & 42 & 2,07 & 0,677 & \\
\hline \multirow[t]{2}{*}{ Sexo } & Feminino & 79 & 1,91 & 0,737 & \multirow{2}{*}{$\begin{array}{l}(t=-0,371 \\
p=0,711)\end{array}$} \\
\hline & Masculino & 11 & 2,00 & 0,775 & \\
\hline \multirow[t]{3}{*}{ Profissão } & Médico veterinário & 72 & 7,68 & 0,601 & \multirow{3}{*}{$\begin{array}{l}(F=0,779 \\
0,462)\end{array}$} \\
\hline & Enfermeiro veterinário & 13 & 7,54 & 0,519 & \\
\hline & Auxiliar & 5 & 7,40 & 0,548 & \\
\hline
\end{tabular}

Analisando individualmente o tipo de acidente laboral, encontrou-se uma relação estatisticamente significativa que associa a ocorrência de corte ou picada com objetos potencialmente contaminados aos indivíduos mais experientes (V Cramer=0,253; $p=$ $0,023)$ e mais velhos ( $V$ Cramer $=0,253 ; p=0,024)$. A literatura realça que trabalhadores 
mais experientes, por estarem mais à vontade e relaxados, têm por vezes menos cuidado; por outro lado, também está descrito que os mais inexperientes se precipitam com mais facilidade e têm atitudes que potenciam a probabilidade e/ou ocorrência de sinistros. No entanto, nesta amostra, convém relembrar que maior experiência implica mais anos de trabalho e, por isso, mais acidentes em número absoluto no total, ainda que sem maior frequência percentual.

Quanto à distribuição de acidentes versus profissão, encontrou-se uma relação estatisticamente significativa entre ser-se Médico Veterinário e o corte/ picada, tal como inserido na Tabela 6- o que se pode justificar dado serem estes profissionais os que mais utilizam agulhas e outros objetos com capacidade de corte. Também aqui se pode concluir que as alíneas inseridas no questionário não esgotaram as respostas, uma vez que quase um quinto dos indivíduos referiu ter tido outros acidentes para além dos já listados.

Tabela 6- Distribuição dos acidentes de trabalho em função das profissões

\begin{tabular}{|c|c|c|c|c|}
\hline Fator/ \% & Médico & Enfermeiro & Auxiliar & $\begin{array}{l}\text { Teste de } \\
\text { Fisher }\end{array}$ \\
\hline $\begin{array}{l}\text { Ter algum acidente de trabalho, } \\
\text { globalmente }\end{array}$ & 94,4 & 92,3 & 80,0 & $\begin{array}{l}x^{2}=2,389 \\
p=0,218\end{array}$ \\
\hline $\begin{array}{l}\text { Corte/ picada com objetos } \\
\text { contaminados }\end{array}$ & 81,9 & 61,5 & 40,0 & $\begin{array}{l}x^{2}=6,269 \\
p=0,039\end{array}$ \\
\hline Mordidela/ arranhadela & 81,9 & 84,6 & 80,0 & $\begin{array}{l}x^{2}=3,491 \\
p=0,157\end{array}$ \\
\hline Acidente de viação & 2,8 & 0,0 & 0,0 & $\begin{array}{l}x^{2}=0,929 \\
p=1,000\end{array}$ \\
\hline Outros acidentes & 20,8 & 23,1 & 0,0 & $\begin{array}{l}X^{2}=0,917 \\
p=0,774\end{array}$ \\
\hline
\end{tabular}

Na sequência dos acidentes de trabalho, os indivíduos foram inquiridos se surgiram limitações laborais; a grande maioria negou tal situação (Gráfico 2), sendo escassos os casos com maior gravidade subjetiva, ou seja, avaliada pelo próprio.

Gráfico 2 - Existência de limitações laborais após o acidente

Relativamente à ocorrência de limitações após o acidente, o teste de Kruskal-Wallis não demonstrou diferenças estatisticamente significativas entre as profissões. De igual modo, o teste $U$ de Mann-Whitney não encontrou qualquer significância estatística em função da idade $(U=475,5 ; p=0,940)$, da experiência profissional $(U=438,5 ; p=0,558)$ e do sexo $(U=453 ; p=0,563)$. Seria de esperar que indivíduos com mais idade tivessem menos condições fisiológicas para recuperar sem limitações dos sinistros. Por outro lado, o acidente referenciado poderia ser mais antigo, dando ao organismo mais tempo para recuperar/ adaptar, versus um sinistro mais recente. 


\section{Atividade laboral e doenças profissionais}

Analisando as principais queixas físicas decorrentes da atividade profissional, 73,3\% referiu ter sintomas. Destacam-se a lombalgia (50\%) e a cervicalgia $(43,3 \%)$, ainda que tenham ficado uma diversidade de sintomas por validar $(34,4 \%)$ reunidos no parâmetro "outras queixas", que não foram tratadas estatisticamente por não existir espaço para serem relatadas por extenso na mesma pergunta (Gráfico 3).

Gráfico 3 - Principais sintomas resultantes da atividade profissional

Em relação à idade, o teste de Qui Quadrado não comprovou qualquer associação estatística significativa com a existência de sintomas eventualmente associados ao trabalho $\left(X^{2}=0,146 ; p=0,812\right)$, facto que não corrobora com o que está descrito na bibliografia, pois as doenças profissionais e não profissionais são mais frequentes à medida que a idade avança, pelo que se esperariam diferenças estatisticamente significativas neste ponto. Realça-se ainda, como fator agravante, que os trabalhadores menos experientes assinalaram com maior probabilidade a existência de sintomas associados ao seu trabalho, ainda que essa diferença não fosse estatisticamente significativa $\left(X^{2}=2,376 ; p=0,123\right)$. Mais uma vez, podem estar aqui envolvidas muitas variáveis que condicionam estes resultados, como personalidade, acontecimentos de vida, ambiente laboral (salubridade, segurança, atitude de chefias e colegas), entre outros.

A ocorrência de sintomas eventualmente associados à atividade laboral foi, no entanto, estatisticamente significativa para o sexo feminino $\left(X^{2}=4,981 ; p=0,026\right)$, destacando-se as maiores diferenças, ainda que não estatisticamente significativas, ao nível da cervicalgia $\left(X^{2}=3,228 ; p=0,072\right)$ e da lombalgia $\left(X^{2}=2,589 ; p=0,108\right)$.

Quanto à distribuição de sintomas pelo tipo de profissão, o teste de Fisher não encontrou diferenças estatisticamente significativas $\left(X^{2}=0,213 ; p=1,000\right)$; ainda assim as respetivas distribuições percentuais podem ser consultadas na Tabela 7. Contudo, facilmente se percebe que as alíneas inseridas no questionário não esgotaram as respostas, uma vez que mais de um terço dos profissionais referiu ter outros sintomas para além dos já mencionados. Em função da literatura e da experiência clínica dos autores seria de esperar que postos de trabalho com mais tarefas físicas apresentassem mais sintomas músculo-esqueléticos, por exemplo.

Tabela 7- Distribuição dos sintomas eventualmente associados ao trabalho, em função das profissões

Fator / \%

Sintomatologia associada ao trabalho

Cervicalgia
Médicos Enfermeiros Auxiliares Teste de Fisher

$\begin{array}{llll}72.2 & 76,9 & 80,0 & \begin{array}{l}X^{2}=0,213 ; \\ p=1,000\end{array} \\ 41,7 & 38,5 & 80,0 & \begin{array}{l}X^{2}=2,768 ; \\ p=0,298\end{array}\end{array}$




\begin{tabular}{lcccl}
\hline Dorsalgia & 34,7 & 30,8 & 0,0 & $\begin{array}{l}\mathrm{X}^{2}=2,240 ; \\
\mathrm{p}=0,362\end{array}$ \\
\hline Lombalgia & 45,8 & 61,5 & 80,0 & $\begin{array}{l}\mathrm{X}^{2}=2,832 ; \\
\mathrm{p}=0,209\end{array}$ \\
\hline Dor nas mãos e dedos & 20,8 & 23,1 & 20,0 & $\begin{array}{l}\mathrm{X}^{2}=0,283 ; \\
\mathrm{p}=1,000\end{array}$ \\
\hline Outros sintomas & 34,7 & 30,8 & 40,0 & $\begin{array}{l}\mathrm{X}^{2}=0,309 ; \\
\mathrm{p}=1,000\end{array}$
\end{tabular}

Por último, analisando a perceção individual relativa à existência de doença(s) associada(s) à atividade laboral, 38,9\% respondeu afirmativamente. Neste caso, destacam-se as referências alusivas a alterações músculo-esqueléticas $(26,7 \%)$ Gráfico 4.

É de realçar que apenas um caso foi declarado como suspeita de doença profissional, estando a aguardar avaliação e parecer final.

Gráfico 4 - Doenças profissionais referenciadas

Nesta amostra, os trabalhadores mais jovens referiram com maior regularidade, terem desenvolvido uma doença ocupacional, embora a diferença não seja estatisticamente significativa $\left(X^{2}=2,927 ; p=0,406\right)$; o mesmo aconteceu com os trabalhadores menos experientes $\left(X^{2}=0,334 ; p=0,563\right)$. $O$ facto de trabalhar há menos tempo poderá contribuir para uma maior valorização dos riscos, face à banalização que poderá surgir com o número de anos de atividade profissional, associados à idade cronológica. Contudo, haverá aqui a considerar outras variáveis como conhecimentos/ experiência adquiridos, personalidade e acontecimentos de vida concretos.

As variáveis sexo e profissão também não aparecem associadas à existência de doenças profissionais, ainda que estas sejam, percentualmente, mais prevalentes entre os auxiliares, talvez por um contato mais próximo e contínuo com os animais (Tabela 8).

Tabela 8- Distribuição das doenças eventualmente associadas ao trabalho, em função das profissões

\begin{tabular}{lcccc} 
Fator/ \% & Médico & Enfermeiro & Auxiliar & $\begin{array}{l}\text { Teste de } \\
\text { Fisher }\end{array}$ \\
\hline $\begin{array}{l}\text { Doenças eventualmente associadas } \\
\text { ao trabalho }\end{array}$ & 38,9 & 38,5 & 40,0 & $\begin{array}{l}\mathrm{X}^{2}=0,157 ; \\
\mathrm{p}=1,000\end{array}$ \\
\hline Eczema/ dermatite & 12,5 & 7,7 & 20,0 & $\begin{array}{l}\mathrm{X}^{2}=0,873 ; \\
\mathrm{p}=0,679\end{array}$ \\
\hline Urticária & 5,6 & 7,7 & 20,0 & $\begin{array}{l}\mathrm{X}^{2}=2,389 ; \\
\mathrm{p}=0,222\end{array}$ \\
& & & &
\end{tabular}




\begin{tabular}{lcccc}
\hline Zoonose & 2,8 & 0,0 & 20,0 & $\begin{array}{l}\mathrm{X}^{2}=3,680 ; \\
\mathrm{p}=0,217\end{array}$ \\
\hline Rinite & 13,9 & 7,7 & 20,0 & $\begin{array}{l}\mathrm{X}^{2}=0,812 ; \\
\mathrm{p}=0,712\end{array}$ \\
\hline Conjuntivite & 5,6 & 0,0 & 20,0 & $\begin{array}{l}\mathrm{X}^{2}=2,558 ; \\
\mathrm{p}=0,388\end{array}$ \\
\hline Lesão músculo-esquelética & 25,0 & 30,8 & 40,0 & $\begin{array}{l}\mathrm{X}^{2}=1,033 ; \\
\mathrm{p}=0,662\end{array}$ \\
\hline
\end{tabular}

\section{Stress laboral}

O stress emergiu como um fenómeno muito frequente, atingindo a quase totalidade da amostra (96,7\%). A dificuldade em conciliar as vidas, profissional e pessoal $(81,1 \%)$, a insatisfação salarial $(76,7 \%)$, a elevada carga de trabalho $(73,3 \%)$, o conflito com os clientes $(68,9 \%)$, a ausência de progressão na carreira $(67,8 \%)$ e a necessidade de realizar turnos noturnos e fins de semana $(64,4 \%)$ emergiram como os fenómenos mais comuns capazes de induzir stress nos profissionais.

Gráfico 5 - Causas de stress laboral

O teste Exato de Fisher não revelou diferenças estatisticamente significativas entre a idade ou a experiência profissional, em função da existência de stress ocupacional. Por vezes está descrito na literatura que funcionários mais jovens sentem mais stress devido à sua inexperiência laboral e falta de estratégias de adaptação; por sua vez, também é de supor que, em algumas circunstâncias, funcionários com mais idade se sintam menos capazes de desempenhar as suas tarefas laborais e que isso gere mais ansiedade.

Observa-se, no entanto, que a existência de stress laboral parece estar estatisticamente associada ao sexo feminino (V Cramer $=0,309 ; p=0,003$ ).

Relativamente à profissão, não se observam diferenças estatísticas significativas no que diz respeito à consciencialização acerca da existência de stress laboral $\left(X^{2}=3,680\right.$; $p=0,208$ ), ainda que seja percetível que os auxiliares o referem menos, face aos outros profissionais. Infere-se que tal esteja em interação com a personalidade ou com a responsabilidade assumida, pois postos de trabalho com menor responsabilidade e tarefas mais simples têm tendência para gerar menos stress.

Simultaneamente, emergem dois fatores de stress predominantemente associados aos Médicos Veterinários, nomeadamente o conflito com os clientes $\left(X^{2}=9,030 ; p=0,011\right)$ e a elevada carga de trabalho $\left(X^{2}=5,286 ; p=0,056\right)$. Na Tabela 9 podem ser consultadas as percentagens de cada fator de stress, associadas a cada profissão. Dada a elevada percentagem obtida na última alínea, os autores perceberam que não foram exaustivos a descriminar as causas que poderiam gerar stress laboral- também aqui teria sido oportuno criar uma pergunta extra. 
Tabela 9 - Distribuição dos focos de stress associados ao trabalho, em função das profissões

\begin{tabular}{|c|c|c|c|c|}
\hline Fator/ \% & Médico & Enfermeiro & Auxiliar & $\begin{array}{l}\text { Teste } \\
\text { de } \\
\text { Fisher }\end{array}$ \\
\hline O trabalho gera stress? & 97,2 & 100,0 & 80,0 & $\begin{array}{l}\left(X^{2}=3,680\right. \\
p=0,208)\end{array}$ \\
\hline Elevada carga de trabalho & 77,8 & 46,2 & 80,0 & $\begin{array}{l}\left(X^{2}=5,286\right. \\
p=0,056)\end{array}$ \\
\hline $\begin{array}{l}\text { Dificuldade em conciliar a vida } \\
\text { pessoal e profissional }\end{array}$ & 84,7 & 69,2 & 60,0 & $\begin{array}{l}\left(X^{2}=3,659\right. \\
p=0,189)\end{array}$ \\
\hline Conflito com os clientes & 76,4 & 38,5 & 40,0 & $\begin{array}{l}\left(X^{2}=9,030\right. \\
p=0,011)\end{array}$ \\
\hline Conflito com os colegas & 23,6 & 23,1 & 40,0 & $\begin{array}{l}\left(X^{2}=0,992\right. \\
p=0,706)\end{array}$ \\
\hline Conflito com as chefias & 36,1 & 23,1 & 20,0 & $\begin{array}{l}\left(X^{2}=1,055\right. \\
p=0,615)\end{array}$ \\
\hline Progressão na carreira diminuta & 72,2 & 53,8 & 40,0 & $\begin{array}{l}\left(X^{2}=3,664\right. \\
p=0,151)\end{array}$ \\
\hline Salário considerado insuficiente & 80,6 & 61,5 & 60,0 & $\begin{array}{l}\left(X^{2}=3,392\right. \\
p=0,172)\end{array}$ \\
\hline Turnos rotativos & 45,8 & 38,5 & 20,0 & $\begin{array}{l}\left(X^{2}=1,281\right. \\
p=0,552)\end{array}$ \\
\hline $\begin{array}{l}\text { Turnos em feriados e fins de } \\
\text { semana }\end{array}$ & 69,4 & 46,2 & 40.0 & $\begin{array}{l}\left(X^{2}=4,037\right. \\
p=0,142)\end{array}$ \\
\hline Eutanásia & 47,2 & 30,8 & 20,0 & $\begin{array}{l}\left(X^{2}=2,181\right. \\
p=0,353)\end{array}$ \\
\hline Outras questões & 41,7 & 38,5 & 0,0 & $\begin{array}{l}\left(X^{2}=2,387\right. \\
p=0,263)\end{array}$ \\
\hline
\end{tabular}

\section{Recurso à equipa de saúde ocupacional}

Em função do que está registado na Tabela 10 , observa-se que a maioria $(63,2 \%)$ tem consulta de medicina de trabalho pelo menos de dois em dois anos. Contudo apenas $28,9 \%$ tem a perceção do trabalho realizado pelo Técnico de Segurança e somente $26,7 \%$ refere ter tido formação relacionada com os riscos laborais, facto que talvez explique alguns dos resultados explanados anteriormente.

Tabela 10- Utilização dos serviços de saúde ocupacional 
Frequência Percentagem

\begin{tabular}{|c|c|c|c|}
\hline \multirow{2}{*}{$\begin{array}{l}\text { Tem exames com o Médico do Trabalho pelo } \\
\text { menos de } 2 \text { em } 2 \text { anos? }\end{array}$} & Sim & 57 & 63,3 \\
\hline & Não & 33 & 36,7 \\
\hline \multirow{2}{*}{$\begin{array}{l}\text { Apercebe-se da presença ou resultados da } \\
\text { atuação do Técnico de Segurança no seu } \\
\text { trabalho? }\end{array}$} & Sim & 26 & 28,9 \\
\hline & Não & 64 & 71,1 \\
\hline \multirow{2}{*}{$\begin{array}{l}\text { Já teve formação relacionada com os seus } \\
\text { riscos laborais? }\end{array}$} & Sim & 24 & 26,7 \\
\hline & Não & 66 & 73,3 \\
\hline
\end{tabular}

Relativamente à realização de exame médico, o teste de Qui quadrado revelou associações estatísticas significativas, que comprovam que os mais velhos $\left(X^{2}=5,606\right.$; $p=0,018$ ) têm maior probabilidade de usufruir desse serviço, pelo menos, de dois em dois anos. Segundo os autores isso poderá eventualmente significar que a Saúde Ocupacional passa a ser mais valorizada à medida que a idade avança e/ ou simplesmente pelo facto de a lei exigir exames anuais acima dos 50 anos, versus de dois em dois anos entre os 18 e os 50 anos.

Em virtude da maior sintomatologia, poder-se-ia supor que o sexo feminino poderia frequentar os exames de saúde ocupacional de forma mais assídua. No entanto, não se verifica qualquer associação relevante relativamente ao sexo $\left(X^{2}=1,844 ; p=0,316\right)$, facto que também se comprova analogamente com a profissão $\left(X^{2}=1,428 ; p=0,486\right)$, ainda que os auxiliares aparentem estar descriminados neste parâmetro (40,0; 61,5 e 65,3\% para Auxiliares, Enfermeiros e Médicos Veterinários, respetivamente).

Quanto à perceção da atuação do Técnico de Segurança no Trabalho, não se acharam relações estatisticamente significativas com a idade, experiência profissional e sexo. De realçar que o desempenho deste é mais discreto que o do Médico do Trabalho, por não contatar direta e obrigatoriamente com todos os trabalhadores. Curiosamente, foram os auxiliares que assinalaram a sua presença de forma estatisticamente significativa $\left(X^{2}=7,576 ; p=0,013\right)$. Seria de supor que tendo mais conhecimentos de saúde os Médicos Veterinários estariam mais atentos a estas questões e não o inverso, contudo, dadas as caraterísticas do trabalho desses técnicos que os obriga a percorrer toda a instituição e a perceber como são compridas as regras de segurança, talvez sejam os auxiliares os responsáveis pelo seu acompanhamento na instituição.

Por fim, os mais velhos e mais experientes assinalaram mais frequentemente ter tido formação relativa aos seus riscos laborais, ainda que não existam associações estatisticamente significativas. Contudo, poderá aqui ter uma vez mais ocorrido enviesamento com o número de anos de trabalho, uma vez que a questão quantificou prevalência de formação ao longo da vida e não recentemente ou por um prazo definido. Não significa necessariamente que os que têm mais experiência laboral valorizem mais as atividades formativas. 
Também para o sexo e profissão, a distribuição entre os que tiveram ou não formação sobre riscos laborais é muito homogénea, pelo que não poderia existir uma diferença estatisticamente significativa (27,8; 23,1 e 20,0\% para Médicos, Enfermeiros e Auxiliares, respetivamente).

\section{Relações entre as diferentes variáveis dependentes em estudo}

\section{Uso de EPIs}

Analisou-se a existência de relações estatísticas entre o uso de EPIs e a ocorrência de acidentes laborais, doenças profissionais declaradas, frequência das consultas de Medicina do Trabalho, contacto com o Técnico de Segurança no Trabalho e acesso a formação sobre riscos laborais (Tabela 11).

Segundo a literatura existente, o uso mais rigoroso e frequente de EPIs deveria diminuir a incidência e gravidade dos acidentes laborais; exceto se o modelo causar alguma dificuldade que potencia a sinistralidade. Por outro lado, o ter tido mais sinistros também poderá, em função da personalidade do trabalhador, fazer com que este valorize mais os riscos profissionais e, por isso, usar os EPIs de forma mais rigorosa, posteriormente. Nesta amostra, não se encontrou qualquer associação estatisticamente significativa entre o uso dos diferentes EPIs e o número de acidentes, segundo o teste $U$ de MannWithney.

De igual forma, o uso frequente e correto dos EPIs deveria estar relacionado, em função da literatura, a menor prevalência de doenças profissionais, mas o teste de Correlação de Spearman não revelou tal. De qualquer modo esta é uma condição que necessita geralmente de muitos anos de exposição, porventura mais dos que a generalidade dos inquiridos trabalhou neste setor.

Tentando perceber se haveria qualquer correlação entre o uso de EPIs e a frequência dos exames de Medicina do Trabalho, tal não se comprovou, através do Teste $U$ de Mann-Whitney. Cada Médico do Trabalho tem a sua própria metodologia, mas seria de esperar que tendo os exames ocupacionais regulares, os indivíduos ficassem mais sensibilizados para o uso de EPIs.

Recorrendo ao Teste $U$ de Mann-Whitney para averiguar a existência de associação entre a perceção da atuação do Técnico de Segurança no Trabalho e a utilização dos diferentes EPIs, observam-se relações estatisticamente significativas para o uso de viseira e uso dos manguitos. No entanto, tendo em conta que quem mais usa este equipamento foi quem menos referiu contactar com estes profissionais ficam muitas dúvidas relativas a esta associação.

Simultaneamente, o uso de máscara esteve associado estatisticamente à existência de formação relacionada com os riscos ocupacionais. No entanto, em teoria, essa formação deveria relacionar-se com todos os EPIs mas, na realidade, entre inúmeros empregadores, os temas específicos podem ter incidido mais nuns riscos, em detrimento de outros. 
Tabela 11- Relações estatísticas significativas entre o uso de EPIs e as restantes variáveis dependentes

\begin{tabular}{llllll}
$\begin{array}{l}\text { Uso de } \\
\text { EPIs }\end{array}$ & \multicolumn{2}{l}{ Teste de Mann-Whitney } & & & $\begin{array}{l}\text { Teste Rho } \\
\text { de } \\
\text { Spearman }\end{array}$ \\
\cline { 2 - 6 } & $\begin{array}{l}\text { Acidentes } \\
\text { laborais }\end{array}$ & $\begin{array}{l}\text { Acesso a consulta } \\
\text { de Medicina do } \\
\text { Trabalho }\end{array}$ & $\begin{array}{l}\text { Acesso ao } \\
\text { Técnico de } \\
\text { Segurança }\end{array}$ & $\begin{array}{l}\text { Acesso a } \\
\text { Formação }\end{array}$ & $\begin{array}{l}\text { Doença } \\
\text { declarada }\end{array}$ \\
\hline Farda & $p=0,789$ & $p=0,447$ & $p=0,524$ & $p=0,546$ & $p=0,428$ \\
\hline Manguito & $p=0,656$ & $p=0,881$ & $p=0,023$ & $p=0,171$ & $p=0,695$ \\
\hline Viseira & $p=0,263$ & $p=0,819$ & $p=0,009$ & $p=0,079$ & $p=0,604$ \\
\hline Máscara & $p=0,959$ & $p=0,082$ & $p=0,143$ & $p=0,014$ & $p=0,173$ \\
\hline Calçado & $p=0,870$ & $p=0,367$ & $p=0,092$ & $p=0,490$ & $p=0,399$ \\
\hline Galochas & $p=0,607$ & $p=0,418$ & $p=0,669$ & $p=0,374$ & $p=0,548$
\end{tabular}

Para terminar, ao tentar perceber a eventual ligação entre o uso de EPIs e a capacidade de identificação/ valorização dos riscos/ fatores de risco laborais, através do Teste de Correlação de Spearman, constatou-se que quem usa globalmente tal tem maior probabilidade de considerar estar sujeito a riscos pertinentes, ainda que a correlação seja fraca, ou seja, sem significado estatístico. O que faz sentido em função do que está descrito na literatura, ou seja, maior perceção de risco aumenta os comportamentos que visam minorar tal. Analisando os equipamentos caso a caso, surgiu o dado de que a utilização de máscara está estatisticamente associada ao contato com circunstâncias cancerígenas (Rho=0,390; $p<0.001)$, por exemplo.

\section{Declaração de suspeita de Doença Profissional}

Associando esta variável com o acesso às consultas de Medicina do Trabalho e com a Formação sobre riscos ocupacionais, não se encontraram diferenças estatisticamente significativas utilizando o Teste $U$ de Mann-Whitney. Ainda que qualquer médico seja obrigado a declarar suspeita de doença profissional, geralmente são os Especialista em Medicina do Trabalho que estão mais familiarizados com o processo, pelo que seria de esperar que existisse associação entre estas variáveis; contudo, por outro lado, a prevalência de doenças profissionais é baixa na generalidade dos contextos laborais (na nossa amostra apenas se identificou um caso). A formação, por sua vez, ainda que dependente dos temas abordados, deveria alertar mais os trabalhadores para o facto de determinadas patologias estarem eventualmente associadas às tarefas laborais.

\section{Formação relativa aos Riscos Ocupacionais}


Segundo o Teste $\mathrm{T}$, em média, quem teve formação teve mais acidentes laborais $(2,08 \pm 0,83)$ do que quem não teve $(7,71 \pm 0,46)$, embora a diferença não seja estatisticamente significativa $(t=1,253 ; p=0,213)$. Este valor pode ser explicado através dos fatores idade e experiência profissional pois, tal como já foi referido, os mais velhos e mais experientes, pelo tempo de contacto com a profissão estiveram expostos a um maior número de acidentes pessoais e também, tendencionalmente, têm maior experiência de contacto com formações sobre riscos profissionais. Dessa forma, acreditando que a formação é eficaz na redução dos riscos e acidentes de trabalho, é possível explicar a inexistência de relação estatística relevante.

Simultaneamente, observa-se que quem teve formação neste contexto identificou, em média, mais riscos/ fatores de risco laborais, embora as diferenças também não sejam estatisticamente significativas $(t=0,621 ; p=0,536)$. Através do teste $U$ de Mann-Whitney, também não foi possível identificar diferenças estatisticamente relevantes com a gravidade atribuída a esses riscos/ fatores de risco.

\section{Gravidade dos riscos/ fatores de risco laborais}

Indivíduos que referiram ter algum acidente de trabalho demonstraram-se mais capazes de valorizar alguns riscos laborais, embora de forma estatisticamente não significativa, a confiar no Teste U de Mann-Whitney, o que está em concordância com o que está descrito na literatura.

De igual forma também se percebeu que trabalhadores com limitações pós-sinistro também valorizavam mais os seus riscos laborais, ainda que sem diferenças significativas, usando o Teste de Correlação de Spearman, o que está em sintonia com o descrito pelos investigadores do tema.

Também existiu situação semelhante para funcionários com doenças profissionais declaradas ou reconhecidas, sobretudo em relação às cargas/ esforço físico, segundo o Teste U de Mann- Whitney, de igual forma, sem surpresa face à experiência clínica dos autores.

\section{PRINCIPAIS LIMITAÇÕES IDENTIFICADAS PELOS AUTORES}

Em algumas questões os autores, com base na bibliografia consultada, enumeraram as diversas respostas, finalizando com uma alínea de "outras"; contudo, esta, em algumas perguntas, obteve valores razoavelmente elevados, facto que justificaria a inclusão de uma outra questão aberta para registar essa informação. No entanto, tal também aumentaria o tamanho do questionário e poderia diminuir a motivação em responder.

Quanto aos EPIs, na versão final foi apagada acidentalmente a alínea onde se mencionavam as luvas, eventualmente o segundo EPI mais usado, supõem os autores, em função da sua experiência clínica. 
Para além disso, a amostra pequena poderá não permitir generalizar as conclusões (sobretudo para os Enfermeiros e Auxiliares de veterinária) e nada poderá garantir que os participantes trabalhavam nos postos que assinalaram e que responderam com sinceridade e coerência. Os autores decidiram realizar o tratamento estatístico dos dados após cerca de oito meses porque o grande volume de respostas ocorreu nos primeiros dias, ou seja, nos últimos meses apenas surgiram mais dois questionários, pelo que inferiram que não seria de esperar muitas mais respostas futuramente.

Há que realçar também que a amostra pequena e o número elevado de respostas possíveis na generalidade das questões pode ter eventualmente contribuído para a obtenção de poucas relações estatisticamente significativas, ainda que a ausência das mesmas, desde que real e não enviesada pela forma como a questão foi colocada, são tão válidas quanto as primeiras.

\section{CONCLUSÃO}

De forma muito sumária pode-se salientar que a amostra incluída neste estudo é jovem, maioritariamente do sexo feminino, com poucos anos de trabalho no setor e tendo como profissão predominante a Medicina Veterinária. A nível de saúde laboral valoriza e identifica bem os riscos/ fatores de risco laborais, usa com regularidade os EPIs, está frequentemente exposta a acidentes de trabalho (ainda que sem limitações na generalidade dos casos) e refere diversos sintomas genéricos que associa ao trabalho, com destaque para o stress.

Entre as diversas relações estatísticas evidenciadas destacam-se, as associações entre o sexo feminino, o stress e a existência genérica de sintomas associados ao trabalho, assim como as associações entre exercer como Médico Veterinário, sentir stress laboral por conflito com os clientes e estar exposto a acidentes por corte/ picada com objetos potencialmente contaminados.

No entanto, tendo em conta o tamanho da amostra, a existência de três classes profissionais de caraterísticas diversas e de questões que não conseguiram abarcar todas as respostas possíveis, considera-se que é possível a existência de enviesamentos nos resultados finais, uma vez que, em algumas situações, se encontraram relações entre variáveis concordantes com a bibliografia consultada (para a generalidade das profissões e em sintonia com a experiência clínica dos autores), noutros casos ocorreu o oposto. Assim, considera-se que este estudo serviu como ponto de partida para uma primeira caraterização sumária do setor, sendo obviamente desejável um estudo de maior dimensão, com questões mais abrangentes e melhor estruturadas.

\section{CONFLITOS DE INTERESSE, QUESTÕES ÉTICAS E/OU LEGAIS}

Nada a declarar. 


\section{AGRADECIMENTOS}

Os autores agradecem à APMVEAC (Associação Portuguesa de Médicos Veterinários Especialistas em Animais de Companhia) pela divulgação do link com o inquérito entre os seus associados; bem como à Safemed e às empresas prestadoras de serviços de saúde ocupacional Quercia, Securilabor, CSW, Medicisforma e Sim Saúde.

\section{BIBLIOGRAFIA}

Santos M, Almeida A. Saúde Ocupacional aplicada aos Profissionais associados a atividades Veterinárias (auxiliares, enfermeiros e médicos). Revista Portuguesa de Saúde Ocupacional on line. 2016, volume 1, s143-s147.

\section{(1)Mónica Santos}

Licenciada em Medicina; Especialista em Medicina Geral e Familiar; Mestre em Ciências do Desporto; Especialista em Medicina do Trabalho; Presentemente a exercer nas empresas Medicisforma, Servinecra, Serviço Intermédico, Securilabor, CSW e SBE; Diretora Clínica das empresas Quercia e Gliese; Diretora da Revista Portuguesa de Saúde Ocupacional on line. Endereços para correspondência: Rua Agostinho Fernando Oliveira Guedes, 42, 4420-009 Gondomar. E-mail: s_monica_santos@hotmail.com.

\section{(2) Armando Almeida}

Doutorado em Enfermagem; Mestre em Enfermagem Avançada; Especialista em Enfermagem Comunitária; Pós-graduado em Supervisão Clínica e em Sistemas de Informação em Enfermagem; Docente na Escola de Enfermagem (Porto), Instituto da Ciências da Saúde da Universidade Católica Portuguesa; Diretor Adjunto da Revista Portuguesa de Saúde Ocupacional on line. 4420-009 Gondomar. E-mail: aalmeida@porto.ucp.pt.

\section{(3) Catarina Lopes}

Licenciada em Enfermagem, desde 2010, pela Escola Superior de Saúde Vale do Ave. A exercer funções na área da Saúde Ocupacional desde 2011 como Enfermeira do trabalho autorizada pela Direção Geral de Saúde, tendo sido a responsável pela gestão do departamento de Saúde Ocupacional de uma empresa prestadora de serviços externos durante 7 anos. Atualmente acumula funções como Enfermeira de Saúde Ocupacional e exerce como Enfermeira Generalista na SNS24. Encontra-se a frequentar o curso Técnico Superior de Segurança do Trabalho.

4715-028. Braga. E-mail: catarinafflopes@gmail.com 


\section{(4)Tiago Oliveira}

Licenciado em Enfermagem pela Universidade Católica Portuguesa. Frequenta o curso de Técnico Superior de Segurança no Trabalho. Atualmente exerce a tempo inteiro como Enfermeiro do Trabalho. No âmbito desportivo desenvolveu competências no exercício de funções de Coordenador Comercial na empresa Academia Fitness Center, assim como de Enfermeiro pelo clube de futebol União Desportiva Valonguense. 4435-718 Baguim do Monte. E-mail: tiago_sc16@hotmail.com.

Santos M, Almeida A, Lopes C, Oliveira T. Panorama da Saúde Ocupacional existente no setor da Veterinária em Portugal (2017/8). Revista Portuguesa de Saúde Ocupacional on line. 2018, volume 5, S23-S40. DOI: 10.31252/RPSO.25.06.2018 